\title{
Social Layers in Agents' Behavior Evaluation System
}

\author{
Krzysztof Cetnarowicz $^{1}$, Renata Cięciwa ${ }^{2}$, and Gabriel Rojek ${ }^{3}$ \\ ${ }^{1}$ Institute of Computer Science \\ AGH University of Science and Technology \\ Al. Mickiewicza 30, 30-059 Kraków, Poland \\ cetnar@agh.edu.pl \\ ${ }^{2}$ Department of Computer Networks \\ Nowy Saccz School of Business - National-Louis University \\ ul. Zielona 27, 33-300 Nowy Sącz, Poland \\ rcieciwa@wsb-nlu.edu.pl \\ ${ }^{3}$ Laboratory of Computer Science \\ AGH University of Science and Technology \\ Al. Mickiewicza 30, 30-059 Kraków, Poland \\ rojek@agh.edu.pl
}

\begin{abstract}
Behavior evaluation is an approach to a security problem in a multi-agent system that reflects security mechanisms in a human society. The main idea of this approach is behavior evaluation of all agents existing in society that is done autonomously by every agent belonging to that society. All autonomous behavior evaluations have to be collected and processed in order to create a collective decision of a society of agents. This approach reflects security mechanisms existing in a small society in which every human being has enough possibilities to observe and evaluate all other members of the society. This results in large computational complexity. In this paper a modification to behavior evaluation is presented which involves two simple social layers. Social layers are characteristic for more complex and larger societies and could be a means of lower computational complexity.
\end{abstract}

\section{Introduction}

Ethically-social mechanisms play a key role in everyday life of every member in a society. These mechanisms enable to find dishonest and undesirable humans on the basis of continuous observation and evaluation of their behavior, or results of that behavior. In a small society every individual observes and evaluates the behavior of all other observable people. The results of autonomous behavior evaluations form one decision of the whole society e.g. decision to exclude somebody from a group. Security mechanisms in society have the decentralized and distributed character - all individuals make their own autonomous evaluations. The results of those evaluations form one decision of the entire society.

In order to design security mechanisms in a multi-agent system that are similar to those functioning in small human societies, two base problems have to be 
solved. The first problem is the design of evaluation mechanisms with which every agent will be equipped. These mechanisms should enable an agent to evaluate the behavior of another agent functioning in society. The results of an agent's behavior are actions which are perceived in a computer system as objects. These objects registered in a certain period of time create a sequence of actions that could be processed in order to qualify whether it is a good or a bad acting agent in this particular system, in which evaluation takes place. Another problem is management, collecting and processing of results of autonomously made behavior evaluations in order to state if a particular agent, which is possibly variously evaluated by different agents, is generally good or intruder (also called a bad agent).

The solutions of the base problems mentioned in the above paragraph were presented in our earlier work (e.g. 5]). After implementing and testing characteristic of security mechanisms for small societies, 3 problems concerning ethicallysocial approach emerge:

- an agent with undesirable behavior could be unidentified by a society of evaluating agents as bad and in consequence this agent would not be excluded from that society,

- a good agent could be mistakenly treated as an intruder,

- computational complexity of security mechanisms is too high because of the base nature of ethically-social mechanisms.

However, we could limit the disadvantageous phenomena with the use of e.g. actions sampling (as presented in [1]), or earlier results collection (as presented in [2]), some additional mechanisms which are noticed in societies are still possible to use and implement in the ethically-social behavior evaluation. The idea of dividing all members of society into two (or even more) groups called social layers seems very useful. The individuals in one social layer could evaluate the behavior of all members of the society and decide which individual is an intruder. The individuals belonging to the other social layer could not have direct mechanisms to discriminate and remove intruders. Clarifying, the first presented layer will be called the remove layer (because of the possibility of direct removing some bad agents) and the second presented layer will be called the subordinate layer (because this layer does not have a direct impact on intruders' removing).

In the above paragraph only some main assumptions about the idea of social layer are presented. To implement this idea the main criterion for belonging to the remove layer has to be decided. Before presenting those details, the main mechanisms of ethically-social security solutions have to be presented: in Sect. 2 behavior evaluation mechanisms that are built into agents and in Sect. 3 mechanisms of management, collecting and processing of results of behavior evaluations. In Sect. 4 the details of the idea of social layers are presented, which is the main topic of this article. The presented theoretical assumptions are next tested and results of these tests are presented in Sect. 5] The main conclusions of this paper are stated in Sect. 6. 


\section{The Division Profile}

All security mechanisms, which enable an agent to make behavior evaluations are named the division profile. Algorithms of the division profile are inspired by immunological mechanisms of $\mathrm{T}$ cells generation, which enable to detect some anomalies. In a case of behavior evaluation, immunological intruders detection mechanisms have to operate on observed actions made by evaluated agent. This approach is opposite to the one proposed in e.g. 34 in which immunological mechanisms operate on the structure of resources. Another difference between artificial immunology and ethically-social approach is the autonomy of a process (an agent) in a secured system - in artificial immunology approach one detection system is considered for a particular computer system (or sub-system). In ethically-social approach every agent autonomously uses his own instantion of detection mechanisms, what induces the necessity of application of some additional algorithms in order to agree collective decision of all agents.

According to immunological mechanisms of $\mathrm{T}$ cells generation the division profile has three stages of functioning: creation of collection of good (self) sequences of actions, generation of detectors and behavior evaluation stage. In further subsections some key aspects of three mentioned stages are presented. More precise description of the division profile functioning is presented in e.g. 56].

\subsection{Collection of Good Sequences of Actions}

The collection $W$ of good sequences of actions of an agent consists of sequences of actions undertaken by this agent. The length of a sequence is fixed to $l$. Presuming there are stored $h$ last actions undertaken by every agent, own collection $W$ will contain $h-l+1$ elements. An agent in order to generate the collection $W$ should collect information representing actions undertaken by him in the past. But, on the other hand, an agent in order to evaluate behavior of an other agent has to collect information representing actions undertaken by the evaluated agent. So an agent should have information about all actions made in the system. This information is stored in the table of actions, in which every agent is equipped. In the table of actions there are stored last $h$ actions of every visible agent.

\subsection{Generation of Detectors}

The generation of detectors of an agent happens when an agent first 'knows' his last $h$ actions, so after this agent has undertaken $h$ actions. The algorithm of detectors generation uses the negative selection - from set $R_{0}$ of generated sequences of length $l$ those matching with any sequence from collection $W$ are rejected. At the start of presented process set $R_{0}$ contains every possible sequence. Sequence matching means that elements of those sequences are the same. Sequences from set $R_{0}$ which will pass such a negative selection create a set of detectors $R$. 


\subsection{Behavior Evaluation Stage}

Once detectors of an agent have been generated, this agent can evaluate behavior of an other agent. The result of behavior evaluation process of an evaluating agent $a$ is a coefficient attributed to an evaluated agent $k$. This coefficient marked as $m_{a}^{k}$ is a number of counted matches between:

- detectors of the agent $a$ which evaluates behavior,

- sequences of actions undertaken by the agent $k$ (this sequences of actions are taken from the table of actions of the agent $a$ ).

Marking the length of a detector as $l$ and the number of stored actions as $h$, the coefficient $m_{a}^{k}$ is a number from a range $\langle 0, h-l+1\rangle$. The maximum of counted matches is equal $h-l+1$, because every fragment of sequence of actions, which has length equal to the length of a detector, can match only one detector.

\section{Mechanisms of Distributed Evaluations Agreement}

An algorithm of agents evaluations management, collection and processing is used to agree one common decision of all agents, which belong to the remove layer. The difficulty in this agreement is caused by the fact that an agent could be differently evaluated by various agents. The discussion of this problem is presented in [5], in this section are presented only key information essential to discuss the main topic of this article.

Each action undertaken by an agent may cause change of the results of behavior evaluations that are done by other agents in the system. This approach lets us formulate the algorithm of evaluation management as follows: If an agent $k$ belonging to any social layer undertakes an action, a request of evaluation of the agent $k$ is sent to all agents (except the agent $k$ ) in the remove layer, which have direct impact on agent removing.

An agent $a$ in case of receiving a request of evaluation of an agent number $k$ sends back only the coefficient $o_{a}^{k}$ in the range $0 \leq o_{a}^{k} \leq 1$. The coefficient $o_{a}^{k}$ is given by function:

$$
o_{a}^{k}=\left(\frac{m_{a}^{k}}{h-l+1}\right)^{4}
$$

where $h-l+1$ is the maximum of counted matches of agent $a$. The power function of evaluation behavior increases a weight of high coefficient $m_{a}^{k}$ (the exponent was set empirically).

In order to decide if the agent $k$ is in general good or bad the environment uses the algorithm of evaluation's collecting and processing, which consists of following actions:

1. All results of behavior evaluations are stored (that results are sent by agents in response to the request of evaluation of the agent $k$ ). 
2. Gained coefficients are summed and then this sum is divided by the number of agents which got the request of evaluation. If this obtained number is greater than $\frac{1}{2}$ agent $k$ is eliminated.

\section{Social Layers}

The presented work focuses on the idea of two coexisting social layers: the remove layer and the subordinate layer. Agents belonging to the remove layer have direct impact on removing of all agents (of all social layers) existing in the environment. Agents belonging to the subordinate layer can only evaluate behavior, but do not have a possibility of presenting their results in order to remove the agents.

In order to make this idea implementable in our ethically-social security system, the criterion for agents to belong to the remove layer has to be stated. Hypothetically, the criterion could be 'experience' of an agent - agents which have been in the secured system long enough could have the right to evaluate and, on this basis, eliminate other agents from their environment. Another criterion is also possible - only those agents are chosen to the remove layer, which evaluations results mostly conform to the opinion of the whole society of agents. Checking this criterion and changing of agents between social layers can be done permanently after some constant time periods. The short discussion presented here does not include all variants of possible criteria. The research presented in this article focuses on the second mentioned criterion.

Analyzing presented ideas, the algorithm of determination which agent will belong to the remove layer could be presented as follows:

1. During the first $h+1$ constant time periods $\Delta t$ all agents belong to the remove layer.

2. Afterwards, during the next checking_time the agents observe each other's evaluation results. If an agent's opinion is the same as the opinion of the whole society it increases its social rank by 1 point.

3. In each $h+1+n *$ checking_time $(\mathrm{n}=1,2, .$.$) constant time period \Delta t 25$ per cent of agents with the highest social ranks are chosen. Only these agents form the remove layer. The opinions of individuals belonging to this social layer are taken into consideration in the process of distinction between good entities and intruders.

4. The social ranks of all agents are reset. The society of agents acts in accordance with the algorithms of management, collecting and processing of results of behavior evaluations presented in Sect. 3. Nonetheless, in the randomly chosen time periods the whole society is requested to evaluate the behavior of an agent $a$ with the purpose of establishing the social ranks of all agents existing in the society. If it happens that any agent belonging to the remove layer is deleted, an agent from the subordinate layer with the highest social rank is moved to the remove layer.

5. The steps number 3 . and 4 . are repeated.

In the research presented below tests are performed in which checking_time is equal to 10,100 constant time periods $\Delta t$. Moreover, the subordinate layer is 
not relieved from the duty of constant behavior evaluation of all other entities in the society despite the fact that their opinions are not taken into consideration in the process of making decision of an agent removing. Consequently, such an approach to the algorithm presented above does not decrease the computational complexity of the security mechanisms, but lets us choose the agents to the remove layer with a high degree of precision.

\section{Results of Experiments}

A multi-agent system was implemented in order to test the security mechanisms existing in a society divided into social layers. The environment of designed system has two types of resources: type A and type B. Resources are used by agents independently, but refilling of all resources is only possible when every type of resources reaches the established low level. The researched system reflects operations in a computer system which should be executed in couples e.g. opening / closing a file. There are a lot of attack techniques that are limited to only one from a couples (or trios...) of obligatory operations (e.g. SYN flood attack [7]). The simulated system has three types of agents:

- type 50/50 agents - agents which take one unit of randomly selected (A$50 \%, \mathrm{~B}-50 \%$ ) resource in every full life cycle; only this type of agents needs resources to refill its energy (if energy level of a 50/50 agent wears off, this agent will be eliminated)

- type 80/20 agents - agents which take one unit of randomly selected (A$80 \%$, B-20\%) resource in every full life cycle; type 80/20 agents should be treated as intruders because the increased probability of undertaking actions of one type can block the system;

- type 100/0 agents - agents which take one unit of A resource in every full life cycle; type 100/0 agents are also called intruders.

To some degree, the behavior of $80 / 20$ agents is similar to the behavior of $50 / 50$ agents but is undesirable in the secured system like intruders behavior. In all experiments presented here there are initially 80 agents of type 50/50, $10-80 / 20$ agents and $10-100 / 0$ agents. All agents in the system are equipped with the division profile mechanisms with parameters $h=18$ and $l=5$. The simulations are run to 1000 constant time periods $\Delta t$ and 20 simulations were performed. Diagrams presented in the next paragraphs show the average taken from 20 simulations.

In the experiments presented below we compare the results obtained in simulations of a homogeneous society and societies divided into two social layers with different checking_time fixed at 10 constant time periods $\Delta t$ in one case and 100 in the other one.

\subsection{The Phenomenon of Self-destruction}

In particular situations a good agent could be mistakenly treated as an intruder. Such a problem is a consequence of the random choice of undertaken actions. As 
a result, some sequences of actions of good agents can be similar to actions of bad agents. This phenomenon has been named the phenomenon of self-destruction.

Several tests were performed in order to check what is the level of mentioned phenomenon depending on the checking_time of remove layer of agent society. Afterwards, the results of these experiments were compared with the results obtained for a homogeneous society. The diagram in Fig. 1] shows the average number of agents type 50/50 in separate time periods.

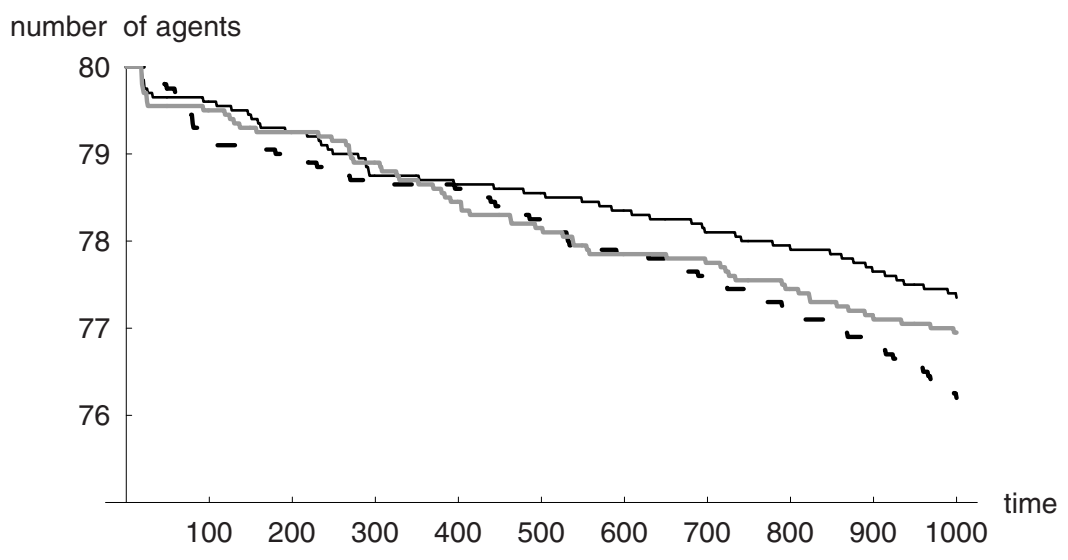

$$
\begin{aligned}
& - \text { homogeneous society } \\
& \ldots \quad \text { stratified society with checking_time }=10 \\
& \text { stratified society with checking_time }=100
\end{aligned}
$$

Fig. 1. Number of type 50/50 agents in separate time periods

In the homogeneous society the level of self--destruction was equal 3,31\% which means that on average not more than 3 good agents were mistakenly treated as intruders and removed from the implemented system. The simulations of the society divided into two social layers showed that the level of the self-destruction phenomenon slightly increased $-4,75 \%$ in case of checking time set to 10 and $3,81 \%$ for checking_time set to 100 constant time periods $\Delta t$. However, the rate of agents' removing tends to be higher during the early stage of the system with checking_time equals 10. Such a problem could stem from the fact that with so short checking_time it is very difficult to differentiate good agents from type 80/20 agents. Therefore, intruders can be chosen to the remove layer and, consequently, have the direct impact on removing of other agents.

The presented research indicate that the social layers have not significant effect on the phenomenon of self-destruction. Nevertheless, the checking_time of the society should be carefully chosen in order to recognize intruders more precisely. 


\subsection{The Rate of Intruders Detection}

In every security system, it is crucial to recognize bad entities as soon as possible and remove them from the environment. In some cases an agent with undesirable behavior could be not identified by a society of evaluating agents as bad and, as a result, this agent would not be excluded from that society.

In our simulations type 100/0 agents were detected during the first 28 constant time periods $\Delta t$. Thus, when the system achieved the behavior evaluation stage all type 100/0 agents were identified properly and eliminated from the system when they tried to undertake actions.
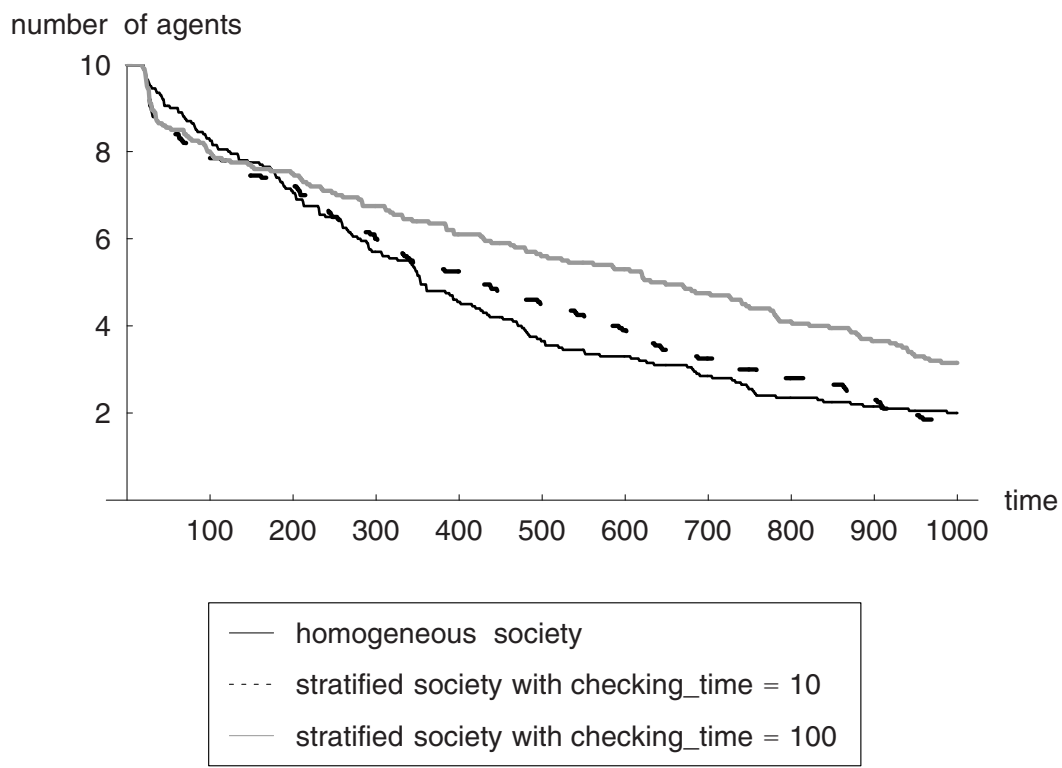

Fig. 2. Number of type $80 / 20$ agents remained in the system in separate time periods

However, the precise recognition of type 80/20 agents is more difficult and takes more time. The division between 50/50 agents and 80/20 agents is hindered by random character of agents decision which resource to undertake (some solutions of this problem were suggested in [26]). The diagram in Fig. 2 shows the average number of agents type 80/20 remained in the system in separate time periods.

In the homogeneous society the level of intruders elimination was equal $80 \%$. The simulations of the society divided into two social layers with checking_time equals 10 showed that this level insignificantly increased to $82,5 \%$. In the case of the stratified society with checking_time equals 100 the level of intruders detection decreased to $68,5 \%$. During the initial stage of simulations the rate of bad agents removing was similar to the results obtained in other mentioned cases. However, this rate seems to be reduced at the moment of dividing the 
society into two social layers. Such a problem could be caused by the fact that the checking_time is too long. Therefore, if the type 80/20 agent hadn't been recognized as an intruder before the division of the society, it could act almost not endangered during the next 100 constant time periods $\Delta t$ due to the fact that the agents, which formed the remove layer probably do not possess detectors to identify its malicious behavior.

\section{Conclusion}

Some modifications in the ethically-social security approach were presented in this paper. The modifications are named social layers, because society of agents is divided into two coexisting groups: remove layer that consist of agents, which can make behavior evaluations and have direct impact on intruder removing and subordinate layer that consists of agents, which do not have direct impact on intruder removing (but can make behavior evaluations). The implementation of idea of social layers presented in the paper contains the criterion which agent should belong to the remove or subordinate layers. The researched criterion is connected with the opinion that only those agents should belong to the remove layer, which behavior evaluations are the closest to all evaluations undertaken in the secured society.

The main field of our interest was how the introducing of social layers would influence on the base problems of the ethically-social security mechanisms. The results of experiments were presented for three cases:

- all agents belong to the remove layer (called in this paper homogeneous society),

- only $25 \%$ of agents belong to the remove layer and the criterion of belonging to this layer is checking every 10 constant time periods $\Delta t$,

- only $25 \%$ of agents belong to the remove layer and the criterion of belonging to this layer is checking every 100 constant time periods $\Delta t$.

The obtained results indicate that the implementation of social layers in ethically-social security system does not have a significant effect on the selfdestruction phenomenon and the rate of intruders detection. However, the time of checking which agent should belong to which layer should be carefully chosen in order to recognize intruders precisely and eliminate them from the system as soon as possible.

To conclude, the idea of social layers seems very interesting because it does not make security mechanisms worse and makes it possible to reduce the computational complexity. The computational complexity could be reduced due to the fact that the agents belonging to the subordinate layer do not have to make behavior evaluations every time, which could be the field of our future research.

\section{Acknowledgments}

This work is partially supported by the Ministry of Science and Higher Education of Poland, grant No. 3 T08B 04229. 


\section{References}

1. Cetnarowicz, K., Cięciwa, R., Rojek, G.: Behavior Evaluation with Actions' Sampling in Multi-agent System. In: Pěchouček, M., Petta, P., Varga, L.Z. (eds.) CEEMAS 2005. LNCS (LNAI), vol. 3690, pp. 490-499. Springer, Heidelberg (2005)

2. Cetnarowicz, K., Cięciwa, R., Rojek, G.: Behavior Evaluation with Earlier Results Collection in Multi-agent System. In: Proceedings of The Agent Days 2005, Malaga, July 7-8, 2005, pp. 77-84 (preprint, 2005)

3. Forrest, S., Perelson, A.S., Allen, L., Cherukuri, R.: Self-nonself Discrimination in a Computer. In: Proc. of the 1994 IEEE Symposium on Research in Security and Privacy, pp. 202-212. IEEE Computer Society Press, Los Alamitos (1994)

4. Hofmeyr, S.A., Forrest, S.: Architecture for an Artificial Immune System. Evolutionary Computation 7(1), 45-68 (2002)

5. Rojek, G., Cięciwa, R., Cetnarowicz, K.: Algorithm of Behavior Evaluation in Multiagent System. In: Sunderam, V.S., van Albada, G.D., Sloot, P.M.A., Dongarra, J. (eds.) ICCS 2005. LNCS, vol. 3516, pp. 711-718. Springer, Heidelberg (2005)

6. Rojek, G., Cięciwa, R., Cetnarowicz, K.: Heterogeneous Behavior Evaluations in Ethically-Social Approach to Security in Multi-agent System. In: Alexandrov, V.N., van Albada, G.D., Sloot, P.M.A., Dongarra, J. (eds.) ICCS 2006. LNCS, vol. 3993, pp. 823-830. Springer, Heidelberg (2006)

7. Schetina, E., Green, K., Carlson, J.: Internet Site Security. Addison-Wesley Longman Publishing Co., Boston (2002) 